\title{
Expression of Brassica napus GLO1 is sufficient to breakdown artificial self-incompatibility in Arabidopsis thaliana
}

Patrick Kenney $^{1,3}$, Subramanian Sankaranarayanan ${ }^{2}$, Michael Balogh $^{1}$, Emily Indriolo ${ }^{1}$

${ }^{1}$ Department of Biology, New Mexico State University, 1200 S. Horseshoe Dr., Las Cruces, NM, 88003, USA

${ }^{2}$ Department of Botany and Plant Pathology, Purdue University, West Lafayette, 47907, Indiana, USA

Current addresses:

${ }^{3}$ University of Missouri, Division of Plant Sciences, Waters Hall 1112 University Ave, Columbia, MO 65201

Corresponding author: Emily Indriolo, indriolo@nmsu.edu, 575-646-2175

ORCID: Emily Indriolo - 0000-0002-7433-1780

Funding: Support for the Indriolo laboratory was provided by university start-up funds provided to Emily Indriolo from NMSU.

Support for undergraduate researcher Patrick Kenney, was provided by the Howard Hughes Medical Institute, Research Scholars Program: \#52008103

\begin{abstract}
:
Members of the Brassicaceae family have the ability to regulate pollination events occurring on the stigma surface. In Brassica species, self-pollination leads to an allele specific interaction between the pollen small cysteine-rich peptide ligand (SCR/SP11) and the stigmatic S-receptor kinase (SRK) that activates the E3 ubiquitin ligase ARC1 (Armadillo repeat-containing 1),
\end{abstract}


resulting in proteasomal degradation of various compatibility factors including Glyoxalase I (GLO1) which is necessary for successful pollination. Suppression of GLO1 was sufficient to reduce compatibility, and overexpression of GLO1 in self-incompatible Brassica napus stigmas resulted in partial breakdown of the self-incompatibility response. Here, we verified if BnGLO1 could function as a compatibility factor in the artificial self-incompatibility system of Arabidopsis thaliana expressing $A l \mathrm{SCR}_{\mathrm{b}}$, AlSRK $\mathrm{b}$ and AlARC1 proteins from A. lyrata. Overexpression of $B n$ GLO1 is sufficient to breakdown self-incompatibility response in A. thaliana stigmas, suggesting that GLO1 functions as an inter-species compatibility factor. Therefore, GLO1 has an indisputable role as a compatibility factor in the stigma in regulating pollen attachment and pollen tube growth. Lastly, this study demonstrates the usefulness of an artificial self-incompatibility system in A. thaliana for interspecific self-incompatibility studies.

Keywords: self-incompatibility, pollination, Arabidopsis, Brassica, interspecific, Glyoxalase I, pollen-pistil interactions

\section{Introduction:}

Many lineages of flowering plants have developed self-incompatibility systems to prevent inbreeding and encourage genetic diversity through outcrossing (Charlesworth and Vekemans 2005; de Nettancourt 2001; Hiscock and Allen 2008; Iwano and Takayama 2012). In the mustard family of plants, which includes the model dicot, Arabidopsis thaliana, Brassica napus (canola) and other economically important species of Brassica, most of the species have a selfincompatibility system to reject 'self' pollen. In the Brassicaceae, the self-incompatibility system is sporophytic, where the recognition occurs between the diploid male and female parts of the 
plant. Acceptance is mediated by the stigma, a dry type, which will only allow the transfer of water from the stigmatic papillae cells to the desiccated pollen grain if it is compatible (HeslopHarrison 1979). A rejected 'self' pollen grain will be unable to hydrate and germinate on the stigma surface as a result of the activation of the self-incompatibility response. One of the major exceptions is A. thaliana, a species that has lost its self-incompatibility system (Boggs et al. 2009; Castric et al. 2014; Indriolo et al. 2012; Schierup et al. 2006; Tsuchimatsu et al. 2010). Thus, the study of this self-incompatibility system has been predominantly focused on Brassica spp and Arabidopsis lyrata (Indriolo et al. 2012; Ivanov et al. 2010; Iwano and Takayama 2012; Kusaba et al. 2001; Schierup et al. 2006; Tantikanjana et al. 2010).

Through the analysis of Brassica spp, the critical components of this self-incompatibility (SI) signaling pathway have been determined. These include the pollen coat ligand Small Cysteine Rich, SCR, or S-locus Protein 11, SP11 (referred to as SCR from here on), the female receptor kinase S-locus Receptor Like Kinase (SRK) which can recognize SCR (Kachroo et al. 2001; Shimosato et al. 2007; Tantikanjana et al. 2010). SRK becomes phosphorylated and activated, and with the cytoplasmic receptor like kinase, M-locus Protein Kinase (MLPK), these kinases phosphorylate and activate downstream signaling components (Kakita et al. 2007; Murase et al. 2004; Samuel et al. 2008). The Armadillo Repeat Containing 1 (ARC1) E3 ubiquitin ligase is one of the proteins phosphorylated and activated by SRK and MLPK in this signaling pathway (Gu et al. 1998; Stone et al. 2003; Stone et al. 1999). ARC1 has been shown to target several compatible pollination factors that are involved in the basal compatibility response including, EXO70A1, Phospholipase D1, (PLD1) and B. napus Glyoxalase 1 (BnGLO1) for ubiquitination (Samuel et al. 2009; Sankaranarayanan et al. 2017; Sankaranarayanan et al. 2015; Scandola and Samuel 2019). 
EXO70A1, a member of the exocyst complex, mediates the delivery of multi-vesicular bodies (in Brassica spp) and secretory vesicles (in A. lyrata and A. thaliana) to the pollen contact point that result in pollen grain hydration (Indriolo et al. 2014; Safavian and Goring 2013; Safavian et al. 2015; Samuel et al. 2009). PLD1 activity during compatible pollination promotes vesicle fusion at the membrane, facilitating exocytosis that is essential for pollen germination (Scandola and Samuel 2019). Glyoxalases (GLO) are enzymes involved in detoxification of methylglyoxal (MG), a cytotoxic byproduct of glycolysis (Sankaranarayanan et al. 2017). BnGLO1 prevents the buildup of cytotoxic methylglyoxal (MG) within the stigmatic papilla cells in response to a 'self' pollen (Sankaranarayanan et al. 2015). BnGLO1 was clearly shown to be a target of ARC1 for ubiquitination, and was more likely to be ubiquitinated when the protein had a MG modification as a part of the increase in MG within the papilla following perception of a 'self' pollen grain and the activation of the self-incompatibility signaling pathway (Sankaranarayanan et al. 2015). Therefore, during a compatible pollination, $B n \mathrm{GLO} 1$ detoxifies MG levels in the stigma and helps to prevent the MG modification of other proteins. During a self-incompatible pollination, the basal pollen response is blocked, including the MG detoxification by BnGLO1. Overexpression of BnGLO1 in self-incompatible $B$. napus stigmas resulted in the breakdown of the selfincompatibility response, causing the acceptance of 'self' pollen (Sankaranarayanan et al. 2015).

Self-incompatibility in $A$. lyrata has been characterized to determine the changes that have occurred between lineage I, including Arabidopsis spp and lineage II, which includes Brassica spp. A. lyrata was also studied to determine the events that lead to the loss of self-incompatibility in the closely related species, A. thaliana (Castric et al. 2014; Indriolo et al. 2012; Nasrallah et al. 2002; Tsuchimatsu et al. 2010). A notable difference from Brassica spp. is that MLPK does not 
appear to play a role in self pollen rejection in A. lyrata (Castric et al. 2014; Indriolo et al. 2012; Nasrallah et al. 2002; Tsuchimatsu et al. 2010). RNAi knock down of ARC1 in A. lyrata sp. patrea resulted in plants that were able to accept incompatible pollen grains and demonstrated that $A R C 1$ is necessary for the rejection of self-pollen in A. lyrata (Indriolo et al. 2012). A survey of over 300 ecotypes of $A$. thaliana showed that all of the accessions had the same $A R C 1$ deletion in their genome indicating that the breakdown of ARC1 in A. thaliana predated the breakdown of SCR and SRK (Castric et al. 2014; Indriolo et al. 2012).

SCR, SRK and ARC1 were demonstrated to have conserved roles in A. lyrata, as observed in Brassica spp. This lead us to the idea that ARC1 could potentially restore a more robust selfincompatibility response in $A$. thaliana through stigma specific expression of the transgene in various ecotypes of $A$. thaliana. Previous studies had reconstructed a self-incompatibility response in $A$. thaliana expressing $A l S C R_{b}$ and $A l S R K_{b}$ alone, but there was a wide range of variability in the robustness of the response based on individual ecotype (Boggs et al. 2009; Nasrallah et al. 2004; Strickler et al. 2013; Tsuchimatsu et al. 2010). Through rigorous surveys of $A$. thaliana ecotypes, some have been identified with functional copies of SRK, such as the Wei-1 and Old-1 accessions. The Wei-1 plants have a functional $S R K_{a}$ and the $S C R_{a}$ gene is inverted; when the inversion was returned to its original state, transgenic Wei-1 plants were able to reject 'self' pollen with the a-haplotype (Tsuchimatsu et al. 2010). With this variation in A. thaliana ecotypes in regards to the retention of functional SRKs and the different phenotypes observed in transgenic $A$. thaliana plants expressing $A l S C R_{b}$ and $A l S R K_{b}$, experiments were performed to look at the role of ARC1 in an artificial transgenic self-incompatibility system. Therefore, two A. thaliana ecotypes were selected: Col-0, which in general did not have a strong self-incompatible phenotype with 
$A l S C R_{b}$ and $A l S R K_{b}$, and the Sha ecotype which did show a self-incompatible phenotype when expressing $A l S C R_{b}$ and $A l S R K_{b}$ (Boggs et al. 2009). In addition to the stigma specific expression of $A l S C R_{b}$ and $A l S R K_{b}$, either $A l A R C 1$ or $B n A R C 1$ were expressed in the stigma under the control of the SLR1 promoter (Indriolo et al. 2014). With the addition of ARC1, a robust self-pollen rejection phenotype was observed in both the Col-0 and Sha ecotypes including aniline blue stains, seed set, and at the ultrastructural level, in the removal of the secretory vesicles likely by autophagy (Indriolo et al. 2014). The generation of self-incompatible A. thaliana with a solid and robust phenotype were a result of the addition of AlARC1 or BnARC1 with AlSCR and AlSRK; these results indicated ARC1 plays a conserved role between lineage I and lineage II of the Brassicaceae (Indriolo et al. 2014). Furthermore, a yeast two hybrid assay demonstrated that BnARC1 and $A l \mathrm{ARC} 1$ could interact with $A l \mathrm{SRK}_{1}$ and $B n \mathrm{SRK}_{910}$ showing that these proteins kept the ability to recognize each other as a part of this signaling pathway despite being phylogenetically separated (Indriolo and Goring 2016).

The role of $B n G L O 1$ has been well defined in $B$. napus self-incompatibility. This poses the question if this compatible stigma factor has a role in Arabidopsis spp. in the artificial selfincompatibility system of $A$. thaliana. To determine if BnGLO1 plays a role in the acceptance of pollen grains, it was transformed into A. thaliana self-incompatibility transgenic lines in the Sha ecotype that contained $A l \mathrm{SCR}_{\mathrm{b}}, A l \mathrm{SRK} \mathrm{b}_{\mathrm{b}}$ and $A l \mathrm{ARC} 1$. By adding stigma specific expression of $B n \mathrm{GLO} 1$ in addition to the endogenous expression, these lines would be similar to a mild over expression line. If the overexpression of $B n \mathrm{GLO} 1$ in addition to the endogenous $A t \mathrm{GLX} 1$ (and likely target of AlARC1) was able to break down the self-incompatibility response it would demonstrate that glyoxalase activity is key to accepting compatible pollen. 


\section{Materials and Methods:}

Plant Materials: Sha wild-type seeds (CS22652), Sha self-incompatible lines $A l \mathrm{ARC} 1+A l \mathrm{SRK}_{\mathrm{b}}+A l \mathrm{SCR}_{\mathrm{b}} \quad$ (Indriolo et al. 2014), and Sha self-incompatible lines $\left(A l \mathrm{ARC} 1+A l \mathrm{SRK}_{\mathrm{b}}+A l \mathrm{SCR}_{\mathrm{b}}\right)$ transformed with $B n \mathrm{GLO} 1$ (Sankaranarayanan et al. 2015) were used in this study.

The wild-type Sha seed stocks came from the Arabidopsis Research Center (ABRC), Columbus, Ohio, USA. All transgenic plants were generated as described in Indriolo et. al. 2014 or in this work.

Plant transformation: All transgenic plants were generated by floral dip as described in Clough and Bent 1997. Sha self-incompatible lines $A l \mathrm{ARC} 1+A l \mathrm{SRK}_{\mathrm{b}}+A l \mathrm{SCR}_{\mathrm{b}}$ plants (Indriolo et al. 2014) were dipped in Agrobacterium tumefaciens containing the BnGLO1 construct (Sankaranarayanan et al. 2015).

Genotyping of transgenic lines: The $A$. thaliana Sha $B n \mathrm{GLO} 1+A l \mathrm{ARC} 1+A l \mathrm{SRK}_{\mathrm{b}}+A l \mathrm{SCR} \mathrm{b}$ were genotyped by PCR (primers in Supplemental Table 1, Supplemental Figure 3), to determine transformants that had all three of the plasmids present in the genome. The 
$A l \mathrm{ARC} 1+A l \mathrm{SRK}_{\mathrm{b}}+A l \mathrm{SCR}_{\mathrm{b}}$ plants were siblings of seeds studied in Indriolo et. al. 2014 which had been previously characterized.

\section{Plant growth conditions:}

Plants were grown under long day conditions; 16 hours light/8 hours dark with a daytime temperature of $22^{\circ} \mathrm{C}$ and a nighttime temperature of $17^{\circ} \mathrm{C}$. A. thaliana seeds were surface sterilized with 1 volume bleach and 2 volumes Triton X-100 for 15 minutes and washed with sterile $\mathrm{dH}_{2} \mathrm{O}$, 4 times. After sterilization, all seeds were stratified for 6 days at $4^{\circ} \mathrm{C}$ and were plated out on $1 / 2$ Murashige \& Skoog, pH 5.8 with 0.4\% w/v phytoblend agar plates. Once the seedlings had 2 true leaves, they were transferred to soil (ProMix-BX + mycorrhizal species, Quebec, Canada). The relative humidity of the chambers was recorded to be between $35-55 \%$ during all experiments.

Pollen adhesion, pollen tube growth and seed set analysis: Pollen grain adhesion and pollen tube growth were observed on freshly opened flowers. To determine the correct age of the freshly opened flowers, stage 12 flower buds (the final bud stage prior to bud opening; (Smyth 1990) from wild-type Sha and transgenic plants were emasculated and covered in plastic wrap to prevent drying of the tissues overnight. 24 hours later, individual anthers from either Sha wild-type flowers or transgenic plants were used to manually apply pollen grains to the stigmas of the emasculated flowers when the papillae were fully elongated. 2 hours-post pollination, whole pistils were removed from the plant and placed in a fixative solution $(300 \mu \mathrm{L}$ of ethanol:glacial acetic acid [3:1]) at room temperature for 30 minutes. Fixative was then removed, and the pistils were washed with sterile $\mathrm{dH}_{2} \mathrm{O}$ three times and then incubated in $300 \mu \mathrm{L}$ of $1 \mathrm{~N} \mathrm{NaOH}$ for $1 \mathrm{hr}$ at $60{ }^{\circ} \mathrm{C}$. Next, 
the $1 \mathrm{~N} \mathrm{NaOH}$ was removed and the pistils were washed with sterile $\mathrm{dH}_{2} \mathrm{O}$ three times and then stained with $300 \mu \mathrm{L}$ of $0.1 \%(\mathrm{w} / \mathrm{v})$ aniline blue at $4^{\circ} \mathrm{C}$ overnight.

To visualize pollen grain adherence and pollen tube penetration, whole pistils were mounted on slides with mounting media $(4 \%(\mathrm{w} / \mathrm{v})$ propyl gallate) to prevent photobleaching. Images were captured with a Zeiss Axio-observer fluorescence microscope. At minimum, 10 pistils were imaged per each individual line for pollen grain adherence (Brightfield) and pollen tube growth (DAPI- 358/463nm). Self-pollen was used in all experiments to determine acceptance or rejection of the pollen on the stigma. All pollination assays were performed at a relative humidity level of between $35-55 \%$ and were monitored with a digital hygrometer in the chamber at all times.

To determine seed set, the number of seeds per silique were tallied for Sha and each independent transgenic line $(\mathrm{n}=10)$. To determine pollen grain adherence to stigmas, the total number of pollen grains were tallied for three different images. The seed set data was graphed and analyzed using R-Studio Version 1.1.463 (2009-2018 RStudio, Inc.). The graph was generated using the ggplot2 package. Statistical analysis included a one-way ANOVA with a Tukey's HSD Post Hoc test from the "agricolae" package. The R script is included in Supplemental Figure 4.

Pollen grain adherence treatments were examined using Welch's ANOVA after running Levene's test for homogeneity. For comparisons between genotypes, we used Dunnett's multiple comparisons test and a Tukey post-hoc test using the SAS for University software package with an $\alpha=0.05$ for a treatment (in this case genotype) to be determined as statistically significant (Cary, 
NC). To determine pollen grain adherence to stigmas, the total number of pollen grains were tallied for three different images.

Confocal imaging of stigmas: To verify expression of BnGLO1 in A. thaliana stigmas, whole pistils were removed from stage 13 flowers. To observe stigmatic papilla cells of the transgenic $A$. thaliana Sha ecotype expressing BnGLO1-RFP, pistils were removed from the flowers and placed in a 24 well plate with $1 \mathrm{~mL}$ sterile $\mathrm{dH}_{2} \mathrm{O}$ containing CellMask Deep Red (Thermo Fischer Scientific) diluted 1:1000. Following incubation for 5 minutes, the solution was removed, the pistils were washed three times with sterile $\mathrm{dH}_{2} \mathrm{O}$ before they were mounted on slides for live cell imaging. Samples were imaged on a Dragonfly 550 spinning disk confocal imaging system mounted on an Olympus IX83 Inverted Microscope using a 30x Apochromat silicon objective (NA 1.05) driven by Andor Fusion software. Images were acquired using an iXon 888 EMCCD camera. Bitplaine Imaris (v9.0.2) software were used to compile and process the images. The final image was generated in Adobe Illustrator.

\section{Expression Profiling \& Protein Sequence Analysis}

We generated an expression heat map for the A. thaliana glyoxalase I gene family using the genevestigator tool (www.genevestigator.com). This analysis examined the relative expression level of the A. thaliana glyoxalase I family in shoot, seedling, stigma, mature pollen, flower, pistil and roots (Hruz et al. 2008). We used The Heat Tree Viewer to analyze the glyoxalase gene family expression in several reproductive tissues and pollen tube growth stages through the pistil. Full length protein sequences for the most closely related copies of Glyoxalase 1 to BnGLO1 were identified by BLAST with the BnGLO1 protein sequence as a query against the translated 
sequences of the $A$. thaliana and $A$. lyrata genomes. A neighbor-joining tree was generated with MEGA X 10.1 software following multiple alignment using Clustal X multiple sequence alignment tool. Multiple alignment of protein sequences of the nearest homologs to BnGLO1 from A. thaliana and with $A$. lyrata were performed using Clustal X and viewed in Jalview. Following sequence alignment, the sequences were trimmed from both the $\mathrm{N}$-terminus and $\mathrm{C}$-terminus to focus on the portion of 163 highly conserved amino acids. Highly conserved amino acids are highlighted in shades of dark gray vs light gray for less conserved residues.

\section{Results:}

\section{Phylogenetic analysis reveals the relationships of Arabidopsis glyoxalase homologs}

The ten most closely related full-length protein sequences from $A$. thaliana and $A$. lyrata were aligned and then used to generate a neighbor-joining tree (Figure 1A). The results show a clear clade where BnGLO1 (magenta box) is sister to Al1g23010 and At1g11840. The next most closely related clade of glyoxalases is that of Atlg67280 and Al2g25800, the two other proteins that were selected to be included in the protein consensus alignment analysis (Figure 1B). Overall, the statistical support based on the bootstrap values for most of the tree is robust with A. thaliana and A. lyrata glyoxalases forming clades from with a single copy of an A. thaliana and A. lyrata glyoxalase at the end of each branch. Only a single copy of each species was instead placed sister to a pair of glyoxalases and with high bootstrap support of 97 for A14g27470 as a single copy with no homologue and support of 98 for At1g80160.

\section{Protein sequence of BnGLO1 is highly conserved among Arabidopsis species}


Our driving question was to examine if the overexpression of $B n$ GLO1 would be able to break down self-incompatibility in $A$. thaliana expressing AlARC1-AlSRK $-A l \mathrm{SCR}_{\mathrm{b}}$; therefore, we aligned the Glyoxalase 1 protein sequences of $A$. thaliana and A. lyrata with that of BnGLO1. This protein alignment (Figure 1B) focuses on the most conserved portion of the full-length protein sequences that correspond to $\mathrm{BnGLO} 1$ amino acids 6-168 of the total protein length of 283 amino acids. The protein alignment shows that this region is conserved as the dark grey regions represent $100 \%$ conservation between the five protein sequences. More interesting are the regions that show more variation; there are two different conserved domains of the more variable portions of the protein sequences. Of the 24 regions, indicated by the white background, 21 of these regions had an amino acid(s) that are conserved between BnGLO1, At1g11840 and Al1g23010, while a different amino acid was identical in the At1g67280 and A12g25800 sequences. Examples of these differences are M13 or F13, T50 or S50 and AVD88-90 or STQ88-90. These differences always change relative to $B n \mathrm{GLO} 1$, with one $A$. thaliana and $A$. lyrata protein sequence matching $B n$ GLO1 while another $A$. thaliana and $A$. lyrata sequence is not conserved with BnGLO1. These points of variation in the conserved protein sequence indicate that At1g11840 and Al1g23010 are likely working as the functional homologues to BnGLO1 and behave as such in each species in respect to protein activity. By using both protein sequence alignment and phylogenetic analysis, we can conclude that BnGLO1 is likely functional similar to that of At1g11840 and A11g23010.

\section{Expression of Glyoxalase 1 in $\boldsymbol{A}$. thaliana tissues}

The expression profile of the most closely related glyoxalases to $B n$ GLO1 were examined in a variety of $A$. thaliana tissues. Figure $1 \mathrm{C}$ shows the results of the expression analysis; each glyoxalase is expressed as the percent of expression potential across tissues; shoot, seedling, 
stigma, pollen, flower, pistil and root. The darker the shade of orange, the higher percent of potential expression (maximum 100\%). Supplemental Figure 2 shows the expression analysis of glyoxalase 1 gene family members in various reproductive tissues including their expression in pistil after various time points of pollination. It is clear that some glyoxalases have a high level of expression in a wide range of tissues such as At 1 g11840, while At2g28420 has a very low level of expression potential in all tissues examined (Figure 1C). At 1 g11840 has fairly robust expression in the stigma, pistil and flower, and reduced expression in mature pollen (Figure 1C). More importantly when compared to the others, this glyoxalase has the closest relation to $B n G L O 1$ and is highly expressed in the stigma and pistil before and after pollination. This indicates a putative role in pollen-pistil interactions (Supplemental Figure 2). Other members that might have a role in pollen-pistil interactions include At1g67280 and Atlg08110. These genes show a moderate level of expression in the stigma and pistils following pollination (Supplemental Figure 2).

Few glyoxalases like Atlg15380, At 1 g80160, At2g3209 and At5g7040 were expressed in the pollen and pollen tubes, indicating that they likely play a role in pollen maturation, pollen tube growth and ultimately in pollen-pistil interactions (Supplemental Figure 2).

\section{Expression of $B n G L O 1$ is stigma specific in transgenic lines}

Three independently transformed lines of Sha $B n \mathrm{GLO} 1+A l \mathrm{ARC} 1+A l \mathrm{SRK}_{\mathrm{b}}+A l \mathrm{SCR}$ were generated by floral dip. All of these lines were first genotyped for the presence of each construct before further characterization (Supplemental Figure $1 \& 3$; Primers listed in Table 1). Only individuals with all of the transgenes were used for further analysis of self-incompatible pollinations compared to Sha wild-type compatible pollinations. 
To verify that the BnGLO1-RFP was expressed in the stigma, unpollinated flowers were imaged using confocal microscopy. To visualize individual papillae, Deep Red CellMask was used to stain the plasma membrane (Figure 2; purple fluorescence). This was used to orient the papillar cells for localization of BnGLO1-RFP in the cells. As previously characterized in B. napus and A. thaliana, the BnGLO1-RFP (Figure 2; red fluorescence) under the SLR1 promoter was expressed in the stigma and it was found both on the plasma membrane and in the cytoplasm (Sankaranarayanan et al. 2017; Sankaranarayanan et al. 2015). These results demonstrate that the transformation and the expression of BnGLO1-RFP into the self-incompatible $A$. thaliana lines was successful.

\section{Self-incompatible lines accept compatible pollen with the overexpression of BnGLO1}

To determine the early pollen response in the Sha $B n \mathrm{GLO} 1+A l \mathrm{ARC} 1+A l \mathrm{SRK}_{\mathrm{b}}+A l \mathrm{SCR}$ plants compared to Sha wild-type and self-incompatible Sha $A l \mathrm{ARC} 1+A l \mathrm{SRK}_{\mathrm{b}}+A l \mathrm{SCR}_{\mathrm{b}}$, aniline blue stains were performed to qualitatively examine pollen grain acceptance in the transgenic stigmas. Wild type self-pollinated stigmas (Figure 3A, Supplemental Figure 1) show pollen grain acceptance and ample pollen tube germination and growth 2 hours post pollination. As previously demonstrated, the Sha $A l \mathrm{ARC} 1+A l \mathrm{SRK}_{\mathrm{b}}+A l \mathrm{SCR}_{\mathrm{b}}$ self-incompatible lines (Fig 3A 1-SI, 2-SI and 5-SI, Supplemental Figure 1) showed a strong self-pollen grain rejection phenotype (Indriolo et al. 2014). Few pollen grains were able to adhere to the stigma and very little pollen tube germination and growth were observed for all three self-incompatible lines. In direct contrast, Sha $B n \mathrm{GLO} 1+A l \mathrm{ARC} 1+A l \mathrm{SRK}_{\mathrm{b}}+A l \mathrm{SCR}_{\mathrm{b}}$ transgenic lines (Fig 3A, 61-SC, 76-SC, 81-SC) appear to be similar to compatible pollen grain acceptance as observed in Sha wild-type stigmas. Pollen tubes germinate readily and penetrate easily at 2 hours after pollination (Fig 3A, 61-SC, 76-SC, 
81-SC Supplemental Fig. 2). Therefore, these images indicate that the addition of BnGLO1 in addition to endogenous $A$. thaliana glyoxalases breaks down the self-incompatibly response.

\section{Self-incompatible lines with BnGLO1 have a compatible pollen response}

To quantitatively characterize the breakdown of the self-incompatibility response, pollen grain adherence and seed set were measured in self-pollinations. Pollen grain adherence clearly demonstrated that the average number of self-pollen grains that were able to adhere to the stigma were statistically different between wild-type compatible pollinations, self-incompatible pollinations and self-incompatible pollinations with BnGLO1 (Figure 3B). Wild-type Sha compatible pollen grain adherence was an average of $102.6 \pm 6.4$ pollen grains per stigma and the self-incompatible lines low pollen grain adherence as previously observed (Figure 3B, Indriolo 2014). Sha $A l \mathrm{ARC} 1+A l \mathrm{SRK}_{\mathrm{b}}+A l \mathrm{SCR}_{\mathrm{b}}$ line 1 had on average $19.2 \pm 6.7$, Sha $A l \mathrm{ARC} 1+$ $A l \mathrm{SRK}_{\mathrm{b}}+A l \mathrm{SCR}_{\mathrm{b}}$ line 2 had on average $31.3 \pm 14.2$ and Sha $A l \mathrm{ARC} 1+A l \mathrm{SRK}_{\mathrm{b}}+A l \mathrm{SCR}_{\mathrm{b}}$ line 5 had on average $22.1 \pm 3.7$ pollen grains adhered per stigma. Statistically similar to Sha wild-type, Sha $B n \mathrm{GLO} 1+A l \mathrm{ARC} 1+A l \mathrm{SRK}_{\mathrm{b}}+A l \mathrm{SCR}_{\mathrm{b}}$ line 61 had on average 88.0 pollen grains \pm 22.3 , Sha $B n \mathrm{GLO} 1+A l \mathrm{ARC} 1+A l \mathrm{SRK}_{\mathrm{b}}+A l \mathrm{SCR}_{\mathrm{b}}$ line 76 had on average $87.3 \pm 12.6$ and the Sha $B n \mathrm{GLO} 1+A l \mathrm{ARC} 1+A l \mathrm{SRK}_{\mathrm{b}}+A l \mathrm{SCR}_{\mathrm{b}}$ line 81 had $80.2 \pm 12.2$ pollen grains on average per stigma. Therefore, the Sha wild-type pollen grains were equivalent to those of the Sha BnGLO1+AlARC1+ $A l \mathrm{SRK}_{\mathrm{b}}+A l \mathrm{SCR}_{\mathrm{b}}$ lines (group a) when analyzed by a Welch's ANOVA followed by a Levine test, while the self-incompatible lines of Sha $A l \mathrm{ARC} 1+A l \mathrm{SRK}+A l \mathrm{SCR}$ were all similar (group b) as summarized in Figure $3 \mathrm{~B}(\mathrm{P}<0.05)$. These results show that the Sha BnGLO1+AlARC1+ $A l \mathrm{SRK}_{\mathrm{b}}+A l \mathrm{SCR}_{\mathrm{b}}$ lines are much closer to Sha wild-type pollen grain adherence implying a loss of the ability to reject self-pollen grains at very early stages of the pollen-pistil interaction. 


\section{Self-incompatible lines expressing BnGLO1 had higher levels of seed set}

Since the self-incompatible lines that were expressing $B n$ GLO1 had similar amounts of pollen grains that were able to adhere to the stigma surface 2 hours post pollination, we were curious to ask if the expression of $B n$ GLO1 lead to increased seed set. Therefore, plants were manually selfpollinated and allowed to develop. Mature siliques were dissected 10 days post-pollination and the total number of seeds per silique were tallied (Figure 4). Sha wild-type plants had an average of $50.2 \pm 2.3$ seeds per silique, while in contrast the Sha $A l \mathrm{ARC} 1+A l \mathrm{SRK}_{\mathrm{b}}+A l \mathrm{SCR}_{\mathrm{b}}$ lines showed much lower number of seeds; line 1 had $9.8 \pm 6.8$ seeds per silique, line 2 had high variance with $20.1 \pm 13.1$ seeds per silique, while line 5 had $18.1 \pm 5.2$ seeds per silique. The Sha $B n \mathrm{GLO} 1+A l \mathrm{ARC} 1+A l \mathrm{SRK}_{\mathrm{b}}+A l \mathrm{SCR}_{\mathrm{b}}$ lines had much higher numbers of seeds per silique; line 61 had $42.1 \pm 6.3$ seeds per silique, line 76 had $40.6 \pm 5.4$ seeds per silique and line 81 had $28.0 \pm 5.3$ seeds per silique.

To determine the significance of these results, these data were examined with an ANOVA followed by a Tukey test $(\mathrm{P}<0.05)$, letters indicate lines that are statically similar e.g. Sha wild-type, Sha $B n \mathrm{GLO} 1+A l \mathrm{ARC} 1+A l \mathrm{SRK}_{\mathrm{b}}+A l \mathrm{SCR}_{\mathrm{b}}$ line 61 and Sha $B n \mathrm{GLO} 1+A l \mathrm{ARC} 1+A l \mathrm{SRK}_{\mathrm{b}}+A l \mathrm{SCR}_{\mathrm{b}}$ line 76. The self-incompatible lines of Sha $A l \mathrm{ARC} 1+A l \mathrm{SRK}_{\mathrm{b}}+A l \mathrm{SCR}_{\mathrm{b}}$ line 1 and Sha $A l \mathrm{ARC} 1+$ $A l \mathrm{SRK}_{\mathrm{b}}+A l \mathrm{SCR}_{\mathrm{b}}$ line 5 were also statistically similar with overlap between line 5 and Sha $A l \mathrm{ARC} 1+A l \mathrm{SRK}_{\mathrm{b}}+A l \mathrm{SCR}_{\mathrm{b}}$ line 2. Interestingly, the self-incompatible line Sha AlARC1+ $A l \mathrm{SRK}_{\mathrm{b}}+A l \mathrm{SCR}_{\mathrm{b}}$ line 2 was determined to be similar to that of Sha BnGLO1+AlARC1+ $A l \mathrm{SRK}_{\mathrm{b}}+A l \mathrm{SCR}_{\mathrm{b}}$ line 81 . These data seem to indicate that the ability of some pollen grains to adhere to the stigma surface is strong enough to overcome the self-incompatibility response, even 
in the Sha $A l \mathrm{ARC} 1+A l \mathrm{SRK}_{\mathrm{b}}+A l \mathrm{SCR}_{\mathrm{b}}$ line 2. Overall, two of the three $B n \mathrm{GLO} 1$ lines were statistically similar to wildtype, thus demonstrating a breakdown or overcoming of the selfincompatibility response.

\section{Discussion:}

Our results demonstrate that the role of GLO1 is conserved between pollen acceptance in B. napus and artificial self-incompatible $A$. thaliana. By using well characterized self-incompatible $A$. thaliana (Indriolo et al. 2014), the addition of BnGLO1 was demonstrated to break down the selfincompatible pollen response in $A$. thaliana. The relationship of $B n$ GLO1 to glyoxalases in $A$. thaliana and A. lyrata was determined using an alignment and generation of a phylogenetic tree based on the full-length protein sequences of the ten most closely related glyoxalases from both Arabidopsis species. The phylogenetic tree revealed that a single copy from both A. thaliana and A. lyrata formed a clade with BnGLO1 and that when these protein sequences were aligned, they were the most highly conserved. Furthermore, At 1 g11840, the most closely related A. thaliana glyoxalase is expressed in the stigma along with other tissues, implying that it may play a role in the stigma during pollinations.

The confocal imaging of unpollinated stigmas demonstrated that BnGLO1-RFP construct localized to the cytoplasm, as was observed when it was expressed in B. napus (Sankaranarayanan et al. 2015). We qualitatively assessed the phenotype of the plants using aniline blue staining to examine self-pollinations and the artificial self-incompatible lines expressing BnGLO1 clearly accepted 'self' pollen grains. Quantitative analysis of pollen grain adherence demonstrated that the $B n \mathrm{GLO} 1$ expressing self-incompatible lines were statistically similar to wildtype via the ANOVA, 
and that the total number of pollen grains was between $79.1-86.2 \%$ of what was observed for wildtype $(=100 \%)$. Therefore, within the first 2 hours following pollination, the BnGLO1 expressing plants are very similar to wildtype and quite different than the self-incompatible lines which showed between $18.8-30.7 \%$ adherence compared to wildtype.

The seed set results overall contribute to the hypothesis that expression of BnGLO1 breaks down the self-incompatible pollination response, with lines 61 and 76 being statistically similar to that of wildtype seed set and had around $83-85 \%$ of the total seed set of wildtype $(=100 \%)$. However, line 81 had a much stronger self-pollen rejection phenotype and was statistically similar to a single self-incompatible line, number 2 having only $56 \%$ of the amount of seeds per silique as wildtype. This amount of variance between transgenic lines is not unusual though; when $B n$ GLO1-RFP was overexpressed in a B. napus W1 (self-incompatible) background, the seed set was not uniform between the three independent lines. One line had an average seed set of 6.5 while the other two were between 1-1.5 seeds per pod and a compatible line had an average of around 29 seeds per pod (Sankaranarayanan et al. 2015). When examined as the percent of a compatible pollination (where 29 seeds $=100 \%$ ) the $B n$ GLO1-RFP overexpression lines were between $3-22 \%$ of wildtype when compared to the seed set of 0 seeds per pod in a W1 self-incompatible pollination (Sankaranarayanan et al. 2015). Considering the behavior of BnGLO1-RFP in B. napus compared to artificial self-incompatible $A$. thaliana, we are not surprised that these are not clear-cut phenotypes; instead these experiments show variation between the phenotypes of different lines. Some of that that variation applies to the fact that the pollen grain adherence of Sha $B n \mathrm{GLO} 1+A l \mathrm{ARC} 1+A l \mathrm{SRK}_{\mathrm{b}}+A l \mathrm{SCR}_{\mathrm{b}}$ line 81 had approximately 81 pollen grains per stigma and was higher than that of Sha $A l \mathrm{ARC} 1+A l \mathrm{SRK}_{\mathrm{b}}+A l \mathrm{SCR}_{\mathrm{b}}$ line 2 that had 31.3 pollen grains per 
stigma, but that pollen grain adherence between these lines did not correspond to the seed set that was observed. Therefore, there may be some additional differences between the artificial selfincompatible lines and our BnGLO1 expressing lines between pollen grain adherence and successful seed set.

Our results indicate that for $B n \mathrm{GLO} 1$ to break down the artificial self-incompatible response in $A$. thaliana, there is a dose dependent aspect of $B n \mathrm{GLO} 1$ in this process. Recent studies have highlighted how the whole genome triplication in Brassica has impacted the function of the regulatory genes in the self-incompatibility pathway in Brassica species. After the whole genome triplication event, most of the self-incompatible signaling pathway genes were maintained as single copy genes in diploid Brassica species. These genes included; ARC1, JDP1, THL1, THL2, Exo70A1, while PLD1 and GLO1 were kept as duplicated genes (Azibi et al. 2020). Further analysis of GLO1 showed that there may be an expression dosage difference involved in part between self-incompatible and self-compatible $B$. rapa cultivars. The self-compatible $B$. rapa var. trilocularis, 'Z1' line when examined for $B n G L O 1$ expression showed expression of the GLO1MF1 (A08) and GLO1-MF2 (A06) genes in stigma cDNA samples. These results show that there are two copies of GLO1 expressed in the stigma of self-compatible B. rapa. In contrast, the $B$. rapa var. pekinensis, 'Chiifu' is a self-incompatible line and only the GLO1-MF1 (A08) copy of GLO1 is expressed. Granted the relative expression level is not equal in the self-compatible ' $\mathrm{Z} 1$ ', between the GLO1-MF1 and GLO1-MF2 in that background but it is present versus absent in the 'Chiifu' B. rapa (Azibi et al. 2020). Our use of expressing an extra copy of BnGLO1 in our selfincompatible $A$. thaliana background may be similar to what is happening between these two 
different $B$. rapa varieties; having more GLO1 activity present in the stigma could help in accepting pollen grains regardless of the activation of the SI response.

These results lead us to propose a model for what is happening in the $A l \mathrm{ARC} 1+A l \mathrm{SRK}_{\mathrm{b}}+A l \mathrm{SCR}_{\mathrm{b}}$ lines compared to the $B n \mathrm{GLO} 1+A l \mathrm{ARC} 1+A l \mathrm{SRK}_{\mathrm{b}}+A l \mathrm{SCR}_{\mathrm{b}}$. In our artificial self-incompatible Sha $A$. thaliana (Figure $5 \mathrm{~A}$ ), the pollen ligand $A l \mathrm{SCR}_{\mathrm{b}}$ is recognized by $A l \mathrm{SRK}_{\mathrm{b}}$. As $A l \mathrm{SRK} \mathrm{b}_{\mathrm{b}}$ is phosphorylated and activated, it in turn activates AlARC1 which targets downstream factors including AtGLX1 for degradation by ubiquitination (Sankaranarayanan et al. 2017; Schmitz et al. 2018). The AtGLX1 levels then decrease and more proteins become MG-modified helping in the self-incompatible pollen response. In contrast, in our $B n \mathrm{GLO} 1+A l \mathrm{ARC} 1+A l \mathrm{SRK}_{\mathrm{b}}+A l \mathrm{SCR} \mathrm{b}_{\mathrm{b}}$ plants (Figure 5B), the self-incompatibility pathway is still activated, but the level of glyoxalase I is much higher than in a normal stigma with the additional expression of the BnGLO1-RFP. Despite AlARC1 being able to ubiquitinate protein targets, there are higher levels of AtGLX1 and BnGLO1-RFP present and they are able to overcome the activity of AlARC1, detoxifying MGmodification of proteins and allowing the stigma to accept the 'self' pollen.

Artificial self-incompatibility in A. thaliana is an ideal system to study and validate compatibility and self-incompatibility genes identified in other related species. BnSCR1 or BnSRK1 have been previously shown to be non-functional in A. thaliana, as expression of BnSCR1-BnSRK1BnARC1 failed to reconstitute self-incompatibility in self-pollinated A. thaliana (Zhang et al. 2019). There are several pieces of evidence that support the idea of differences between Brassica and Arabidopsis self-incompatibility signaling pathways. These observed differences arise due to variations in the upstream components of self-incompatibility signaling (S-haplotype genes) and 
the downstream components of the signaling pathway like ARC1 and interacting partners including EXO70A1, GLO1 and PLD1 are conserved. Previously it has been demonstrated that expression of RFP:BnExo70A1 is able to rescue the stigmatic fertility defect observed in $A$. thaliana exo70A1 mutants suggesting that there is an evolutionarily conserved function for Exo70A1 in the dry stigmas of the Brassicaceae (Samuel et al. 2009).

Despite the divergence of Brassica from Arabidopsis some 20 million years ago, BnGLO1 shares a high sequence similarity to the stigma expressed glyoxalase I homologs in Arabidopsis thaliana (Franzke et al. 2011; Wang et al. 2011). It is not surprising that BnGLO1 could function in $A$. thaliana unlike BnSCR1 and BnSRK1 that show a high degree of variation in their sequences. It would be interesting to see if a reciprocal experiment of transferring Arabidopsis GLXI genes into self-incompatible $B$. napus lines could rescue the SI phenotype. Presence of a large GLX1 gene family (11 members) in A. thaliana and a high functional redundancy makes it difficult to identify their plausible role in pollination as single mutants often fail to exhibit any pollination or fertility phenotypes. Generation of higher order knockout mutants of GLX1 homologues in A. thaliana or stigma specific suppression of multiple $A t \mathrm{GLX} 1 \mathrm{~s}$ using an RNAi approach is required to unequivocally demonstrate the role of GLX1 in mediating compatible pollination in Arabidopsis. Nevertheless, in this study we were able to circumvent the problem of functional redundancy and unmask the functional role of GLO1 in A. thaliana pollination using the artificial selfincompatibility system. Phospholipase D (PLD) is another compatibility factor that exists as a part of a large gene family in Arabidopsis; the same approach can be used to validate its role in pollination by utilizing the $A$. thaliana artificial self-incompatibility system. These proposed experiments will further help us to understand the conservation of self-incompatibility signaling 
mechanisms and components in Arabidopsis and Brassica. In conclusion, this study has validated the functional role of GLO1 as a compatibility factor in Arabidopsis self-incompatibility and our study supports the idea that despite the divergence of upstream self-incompatibility signaling components of Arabidopsis and Brassica, the downstream signaling components are conserved between the two species.

Contributions: SS and EI initiated the project and designed research; PK, SS, MB and EI performed all of the experiments. MB and EI statistically analyzed the results. EI and SS wrote the manuscript. EI obtained funding and supervised the project. All authors discussed the results and commented on the manuscript. The authors would lastly like to thank Ms. Alejandra Cobos and Dr. Marcus Samuel for critical feedback on the manuscript.

\section{Figure legends:}

\section{Figure 1. Expression and Alignment of Glyoxalase 1}

1A.) Phylogenetic analysis of the BnGLO1, Arabidopsis lyrata glyoxalase homologs and Arabidopsis thaliana glyoxalase I family genes. The scale bar represents 0.5 substitutions per amino acid position. Boot strap values are shown at the nodes of each branch of the tree. 1B.) Multiple sequence amino-acid alignment of BnGLO1 with its nearest Arabidopsis thaliana and Arabidopsis lyrata homologs. Highly conserved amino acids are highlighted in shades of dark gray vs light gray for less conserved residues. Alignment is based on BnGLO1 from residue 6-168.

1C.) Microarray expression data of Arabidopsis thaliana Glyoxalase 1 family members (www.genevestigator. com). The darkest shade of orange indicates genes at $100 \%$ of expression potential while the lightest orange shade indicates $0 \%$ of expression potential. 
Figure 2. Localization of BnGLO1-RFP in stigmatic papillae.

Live cell imaging of stage 13 flowers of $A$. thaliana. Sha wild-type stigmas were stained with the CellMask Deep Red stain to visualize the plasma membrane as shown in purple. Sha BnGLO1$\mathrm{RFP}+A l \mathrm{ARC} 1+A l \mathrm{SRK}_{b}+A l \mathrm{SCR}_{b}$ transgenic lines imaged by confocal to visualize $B n \mathrm{GLO} 1-\mathrm{RFP}$ and CellMask Deep Red stain. BnGLO1-RFP is shown in red with the CellMask Deep Red stain in purple for the plasma membrane. Scale bars $=5 \mu \mathrm{m}$.

Figure 3. Self-pollination phenotypes of Sha AlARC1+AlSRK $K_{b}+A l S C R_{b}$ and Sha BnGLO1$R F P+A l A R C 1+A l S R K_{b}+A l S C R_{b}$ stigmas.

3A.) Brightfield and Aniline Blue stains of stigmas. Sha wild-type self-pollinations show the acceptance of self-pollen grains 2 hours post pollination an example of a self-compatible pollination. Scale bar $=50 \mu \mathrm{m}$. 3B.) Average number of pollen grains per stigma 2 hours postpollination. Pollen grain adherence was compared by a one-way ANOVA followed by a Tukey post hoc test $(\mathrm{p}<0.5), \mathrm{n}=3$ stigmas per each transgenic line. Relative Humidity was between 35 $55 \%$ during the pollinations.

Figure 4. Overexpression of BnGLO1 in self-incompatible pistils restores the acceptance of incompatible pollen grains resulting in seed set.

To determine if the expression of $B n G L O 1$ in self-incompatible pistils had a long-term impact on pollen-pistil interactions, flowers were manually self-pollinated and allowed to set seed. 10 to 11 days after manual pollination, siliques were dissected, and the total number of seeds tallied. These data were analyzed with a one-way ANOVA with a Tukey post hoc test $(\mathrm{p}<0.5), \mathrm{n}=10$ siliques 
per each transgenic line. Relative Humidity was between $35-55 \%$ during manual pollinations and subsequent time in the growth chambers.

Figure 5. Model of BnGLO1 action during self-incompatible pollinations.

5A.) During a self-pollination in the artificial self-incompatible A. thaliana, SRK is able to detect SCR on the pollen grain surface. SRK becomes phosphorylated and activated, and in turn phosphorylates ARC1. ARC1 then ubiquitinates GLX1 which decreases due to degradation in the 26S proteasome. 5B.) With the addition of $B n$ GLO1-RFP in the artificial self-incompatible $A$. thaliana, there is more glyoxalase present than normal. Even though SCR is still able to activate SRK and ARC1 activity, the level of GLX1 and BnGLO1-RFP is high enough that ARC1 cannot ubiquitinate all of it for degradation in the $26 \mathrm{~S}$ proteasome. The higher amounts of GLX1 and $B n \mathrm{GLO} 1$ then facilitates the acceptance of the 'self' pollen grain.

Supplemental Figure 1. Additional Aniline Blue stains of A. thaliana stigmas 2 hours post pollination.

Manual self-pollination results of Sha wild-type compatible pollinations, Sha incompatible pollinations of $A l A R C 1+A l S R K_{b}+A l S C R_{b}$ lines and the Sha BnGLO1+AlARC1+AlSRK $+A l S C R_{b}$ lines. Scale bar $=50 \mu \mathrm{m}$.

Supplemental Figure 2. The reproductive expression data series of Arabidopsis thaliana Glyoxalase family members generated with Arabidopsis Heat Tree Viewer.

Supplemental Figure 3. PCR genotyping of all transgenic A. thaliana lines. 
A. thaliana Sha plants were genotyped for the presence of transgenes. Line 61,76 and 81 were the plants that were positive for the presence of AlARC1,AlSCR-AlSRK and BnGLO1 with primers that were specific for each of these constructs. Sizes for each PCR product are included in the figure. The previously characterized A. thaliana Sha artificial self-incompatible lines 1,2 and 5 were confirmed by PCR with the AlARCl and the AlSCR-SRK constructs.

\section{Supplemental Figure 4. R script.}

R script used to analyze seed set and pollen grain adherence.

\section{Table 1. Primers used in genotyping transgenic plants.}

\section{References:}

Azibi T et al. (2020) Impact of whole genome triplication on the evolutionary history and the functional dynamics of regulatory genes involved in Brassica self-incompatibility signalling pathway. Plant Reproduction 33:43-58

Boggs NA et al. (2009) Expression of distinct self-incompatibility specificities in Arabidopsis thaliana. Genetics 182:1313-1321

Castric V, Billiard S, Vekemans X (2014) Trait transitions in explicit ecological and genomic contexts: plant mating systems as case studies. Advances in experimental medicine and biology 781:7-36

Charlesworth D, Vekemans X (2005) How and when did Arabidopsis thaliana become highly selffertilising. Bioessays 27:472-476

de Nettancourt D (2001) Incompatibility and incongruity in wild and cultivated plants. 2nd edn edn. Springer, Berlin

Franzke A, Lysak MA, Al-Shehbaz IA, Koch MA, Mummenhoff K (2011) Cabbage family affairs: the evolutionary history of Brassicaceae. Trends Plant Sci 16:108-116

Gu T, Mazzurco M, Sulaman W, Matias DD, Goring DR (1998) Binding of an arm repeat protein to the kinase domain of the S-locus receptor kinase. Proc Natl Acad Sci U S A 95:382-387

Heslop-Harrison J (1979) An interpretation of the hydrodynamics of pollen. Amer J Bot 66:737743

Hiscock S, Allen A (2008) Diverse cell signalling pathways regulate pollen-stigma interactions: the search for consensus. New Phytologist 179:286-317

Hruz T et al. (2008) Genevestigator v3: a reference expression database for the meta-analysis of transcriptomes Adv Bioinformatics 2008:420747-420747 Indriolo E, Goring DR (2016) Yeast two-hybrid interactions between Arabidopsis lyrata S Receptor Kinase and the ARC1 E3 ligase. Plant Signaling \& Behavior 11:e1188233 
Indriolo E, Safavian D, Goring DR (2014) The ARC1 E3 ligase promotes two different selfpollen avoidance traits in Arabidopsis thaliana. Plant Cell 26 (4) 1525-1543

Indriolo E, Tharmapalan P, Wright SI, Goring DR (2012) The ARC1 E3 ligase gene is frequently deleted in self-compatible Brassicaceae species and has a conserved role in Arabidopsis lyrata self-pollen rejection. Plant Cell 24:4607-4620

Ivanov R, Fobis-Loisy I, Gaude T (2010) When no means no: guide to Brassicaceae selfincompatibility. Trends Plant Sci 15:387-394

Iwano M, Takayama S (2012) Self/non-self discrimination in angiosperm self-incompatibility. Current Opinion in Plant Biology 15:78-83

Kachroo A, Schopfer CR, Nasrallah ME, Nasrallah JB (2001) Allele-specific receptor-ligand interactions in Brassica self-incompatibility. Science 293:1824-1826

Kakita M et al. (2007) Two distinct forms of the M-locus protein kinase localize to the plasma membrane and interact directly with the S-locus receptor kinase to transduce selfincompatibility signaling in Brassica rapa. Plant Cell 19:3961-3973

Kusaba M, Dwyer K, Hendershot J, Vrebalov J, Nasrallah JB, Nasrallah ME (2001) Selfincompatibility in the genus Arabidopsis: characterization of the $\mathrm{S}$ locus in the outcrossing A. lyrata and its autogamous relative A. thaliana. Plant Cell 13:627-643

Murase K, Shiba H, Iwano M, Che FS, Watanabe M, Isogai A, Takayama S (2004) A membraneanchored protein kinase involved in Brassica self-incompatibility signaling. Science 303:1516-1519

Nasrallah ME, Liu P, Nasrallah JB (2002) Generation of self-incompatible Arabidopsis thaliana by transfer of two S locus genes from A. lyrata. Science 297:247-249

Nasrallah ME, Liu P, Sherman-Broyles S, Boggs NA, Nasrallah JB (2004) Natural variation in expression of self-incompatibility in Arabidopsis thaliana: implications for the evolution of selfing. Proc Natl Acad Sci U S A 101:16070-16074

Safavian D, Goring DR (2013) Secretory activity is rapidly induced in stigmatic papillae by compatible pollen, but inhibited for self-incompatible pollen in the Brassicaceae. PLoS One 8:e84286

Safavian D, Zayed Y, Indriolo E, Chapman L, Ahmed A, Goring D (2015) RNA silencing of exocyst genes in the stigma impairs the acceptance of compatible pollen in Arabidopsis. Plant Physiol doi:10.1104/pp.15.00635

Samuel MA, Chong YT, Haasen KE, Aldea-Brydges MG, Stone SL, Goring DR (2009) Cellular pathways regulating responses to compatible and self-incompatible pollen in Brassica and Arabidopsis stigmas intersect at Exo70A1, a putative component of the exocyst complex. Plant Cell 21:2655-2671

Samuel MA, Mudgil Y, Salt JN, Delmas F, Ramachandran S, Chilelli A, Goring DR (2008) Interactions between the S-domain receptor kinases and AtPUB-ARM E3 ubiquitin ligases suggest a conserved signaling pathway in Arabidopsis. Plant Physiol 147:2084-2095

Sankaranarayanan S et al. (2017) Glyoxalase Goes Green: The Expanding Roles of Glyoxalase in Plants. International Journal of Molecular Sciences 18:898

Sankaranarayanan S, Jamshed M, Samuel MA (2015) Degradation of glyoxalase I in Brassica napus stigma leads to self-incompatibility response. Nature Plants 1:15185

Scandola S, Samuel MA (2019) A Flower-Specific Phospholipase D Is a Stigmatic Compatibility Factor Targeted by the Self-Incompatibility Response in Brassica napus. Current Biology 29:506-512.e504 
Schierup MH, Bechsgaard JS, Nielsen LH, Christiansen FB (2006) Selection at work in selfincompatible Arabidopsis lyrata: mating patterns in a natural population. Genetics 172:477-484

Schmitz J, Rossoni AW, Maurino VG (2018) Dissecting the Physiological Function of Plant Glyoxalase I and Glyoxalase I-Like Proteins. Frontiers in Plant Science 9 doi:10.3389/fpls.2018.01618

Shimosato H et al. (2007) Characterization of the SP11/SCR high-affinity binding site involved in self/nonself recognition in brassica self-incompatibility. Plant Cell 19:107-117

Smyth DR, Bowman, J.L., and Meyerowitz, E.M. (1990) Early flower development in Arabidopsis. Plant Cell 2:755-767

Stone S, Anderson E, Mullen R, Goring D (2003) ARC1 is an E3 ubiquitin ligase and promotes the ubiquitination of proteins during the rejection of self-incompatible Brassica pollen. Plant Cell 15:885-898

Stone SL, Arnoldo M, Goring DR (1999) A breakdown of Brassica self-incompatibility in ARC1 antisense transgenic plants. Science 286:1729-1731

Strickler SR, Tantikanjana T, Nasrallah JB (2013) Regulation of the S-Locus Receptor Kinase and Self-Incompatibility in Arabidopsis thaliana. G3: Genes/Genomes/Genetics 3:315-322

Tantikanjana T, Nasrallah ME, Nasrallah JB (2010) Complex networks of self-incompatibility signaling in the Brassicaceae. Current Opinion in Plant Biology 13:520-526

Tsuchimatsu T et al. (2010) Evolution of self-compatibility in Arabidopsis by a mutation in the male specificity gene. Nature 464:1342-1346

Wang X et al. (2011) The genome of the mesopolyploid crop species Brassica rapa. Nature Genetics 43:1035-1039

Zhang T et al. (2019) Generation of Transgenic Self-Incompatible Arabidopsis thaliana Shows a Genus-Specific Preference for Self-Incompatibility Genes. Plants (Basel) 8:570 
$1 \mathrm{~A}$
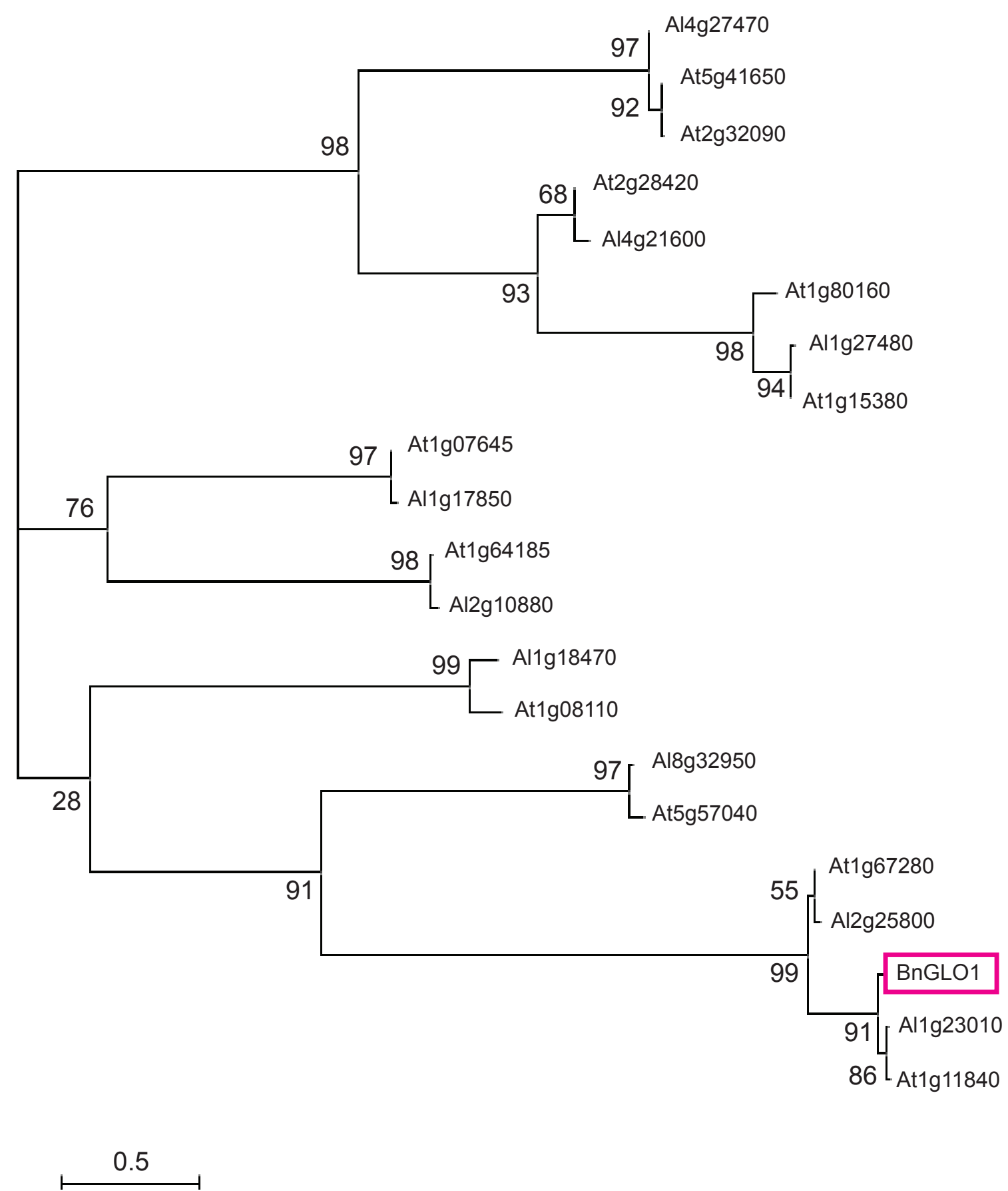


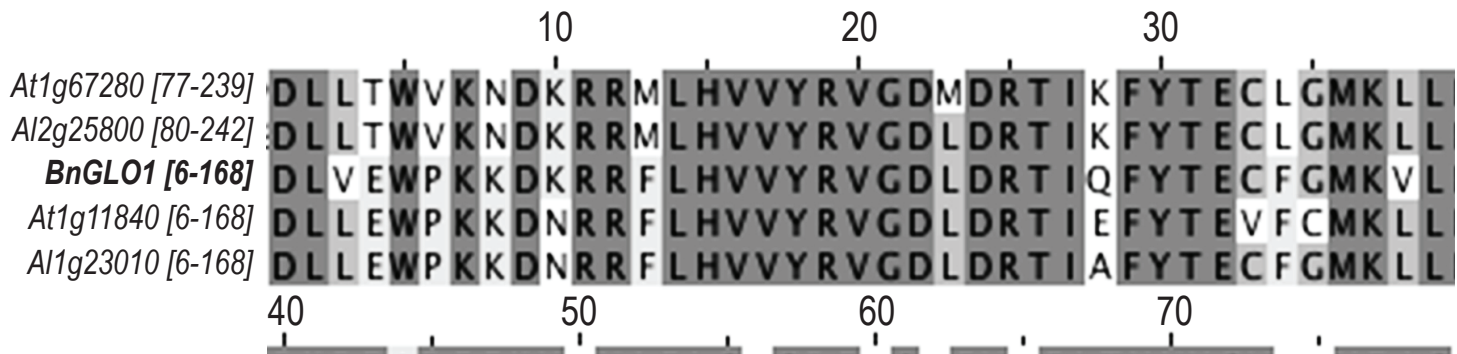

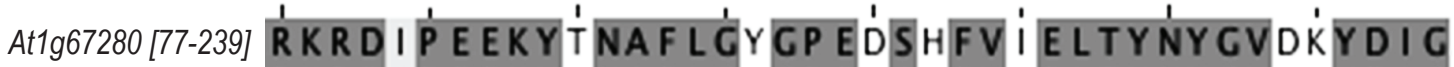
Al2g25800 [80-242] R K R D I P E EKYTNA F L GYGP EDSHFV I E L TYNYGVDKY D I G BnGL01 [6-168] RKR DVP E EKYSNAF L GF GP ETSNFVVE LTYNYGVS SYD I G At1g11840 [6-168] R K R D I P E EKYSNAF L G F GP E T SNFVVE L TYNYGVS SYD I G Al1g23010 [6-168] R KR DVP E EKYSNAF L GF GPETSNFVVELTYNYGVSSYDIG $80 \quad 90 \quad 100 \quad 110$

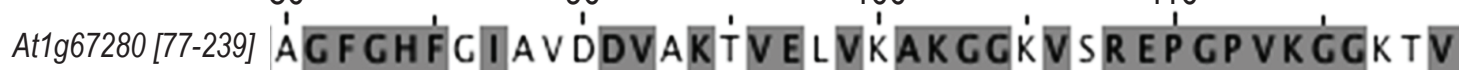
Al2g25800 [80-242] A G F GHFC I AVDDVAK TV E L VKAKGGKVAR EPGPVKGGKTV BnGL01 [6-168] T G F GHFA I S TQDVSKMV EAVRAKGGNVTR EP GPVKGGCSV At1g11840 [6-168] T G F GHFA I S TQDVSK LV ENVRAKGCNVTR EP GPVKGGCSV Al1g23010 [6-168] T G F GHFAISTQDVSK LVETVRAK GCNVSR EP GPVKGCCSV 120 130 140 150

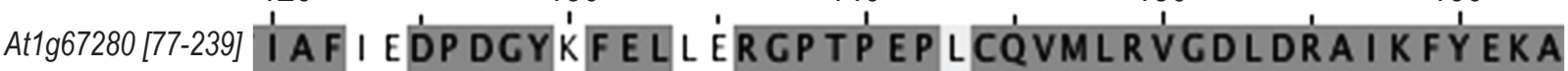
Al2g25800 [80-242] I A F I EDP DGYK F E LLERGP TP EPLCQVMLRVGDLDRA I K FY EKA BnGL01 [6-168] I A FVKDPDGYT F E L I QR GP T P EP LCQVMLRVGDLDRA I K FY EKA At1g11840 [6-168] I A FVKDP DGYT F E L I QR GP T P EP F CQVMLRVGDLDRA I K FY EKA Al1g23010 [6-168] I A FVKDP DGYTIF L I QR GP TPEPFCQVMLRVGDLDRA IKFYEKA

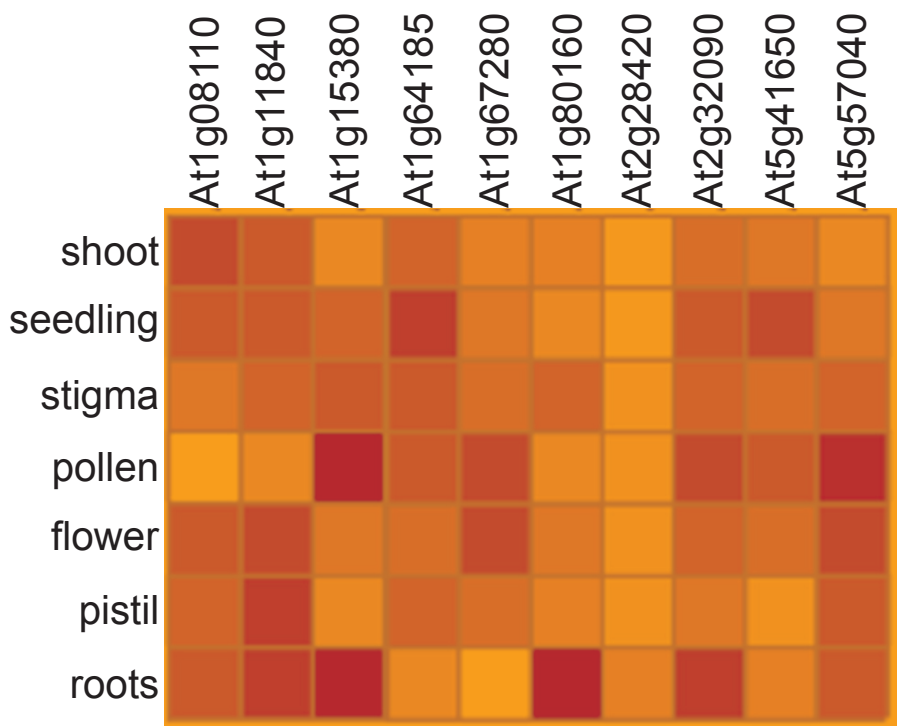

percent of expression potential

$0 \%$ $100 \%$ 
Sha

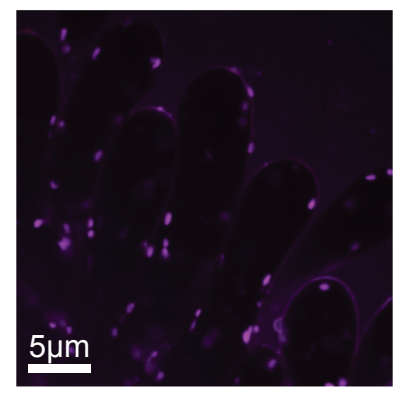

wild-type
Sha $B n G L O 1-R F P+A / A R C 1+A / S R K b+A / S C R b$

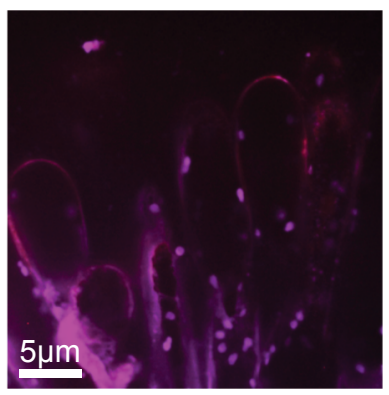

line 61

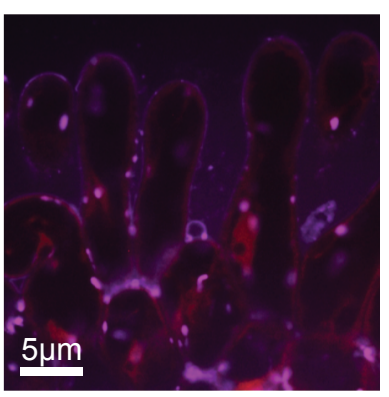

line 76

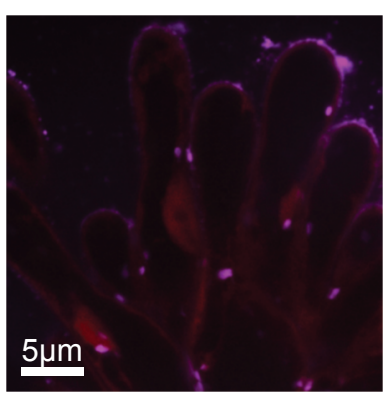

line 81

red - BnGLO1+RFP purple - plasma membrane stain 
Sha wild-type - SC
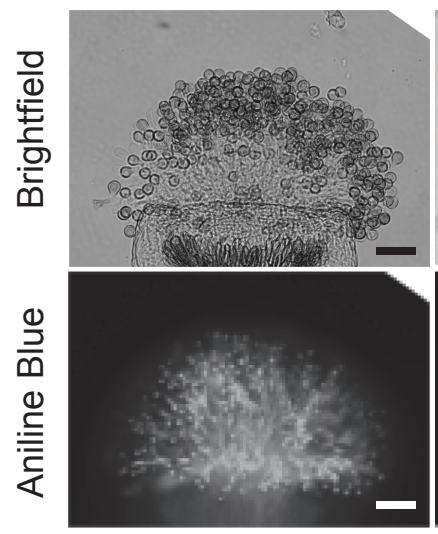

2-SI
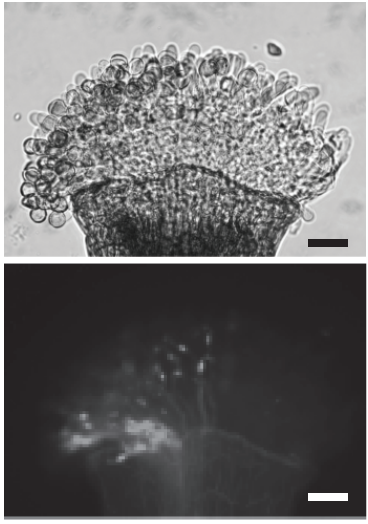
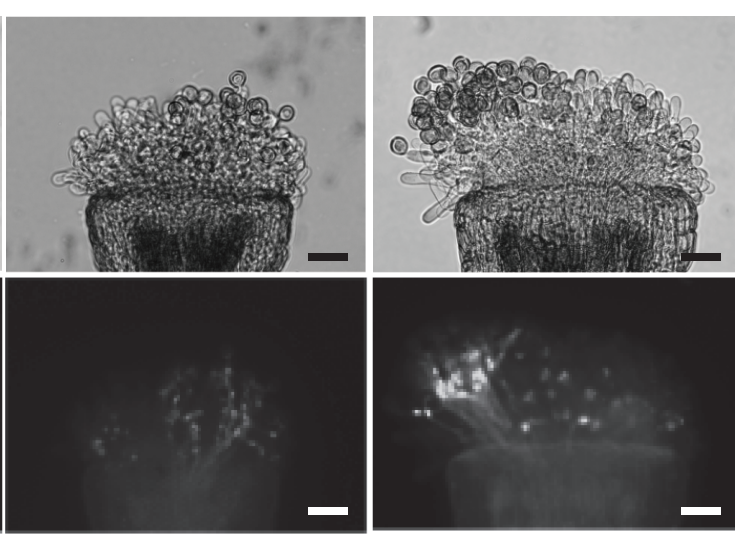

Sha $B n G L O 1+A R C 1+S R K$ lines self pollinated

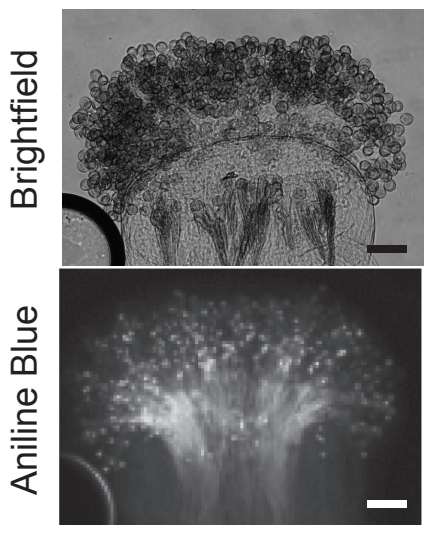

76-SC

81-SC

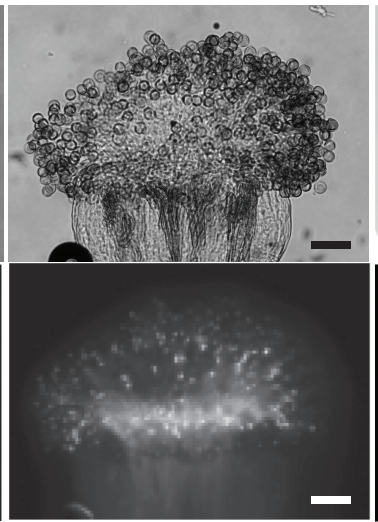

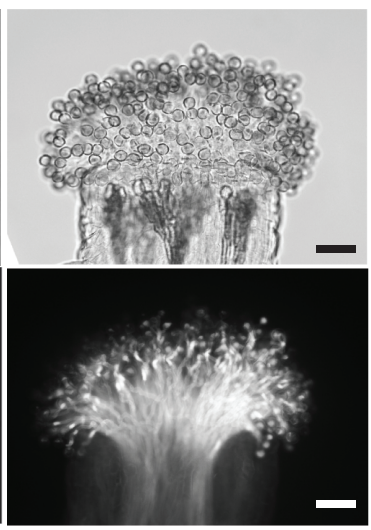

3B

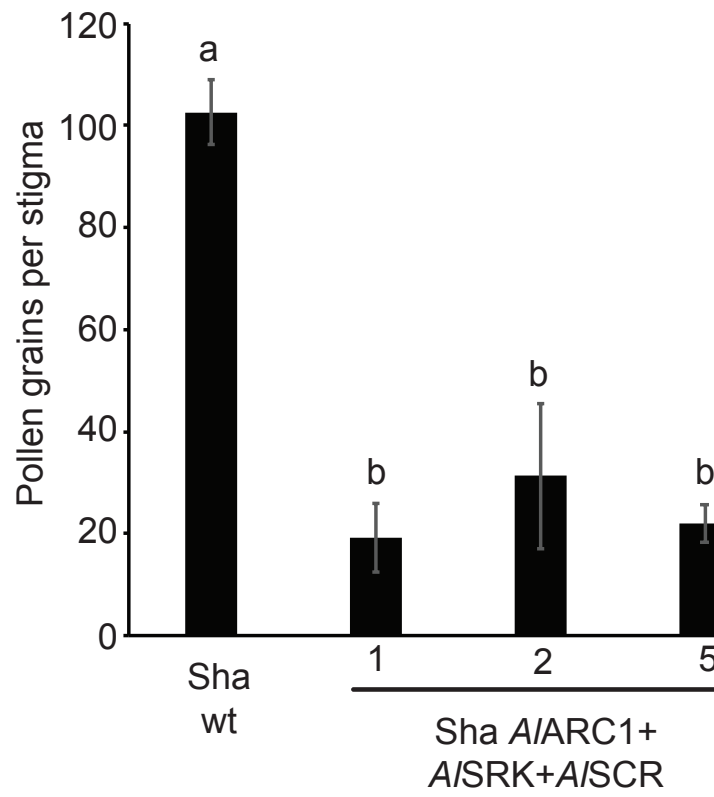

a
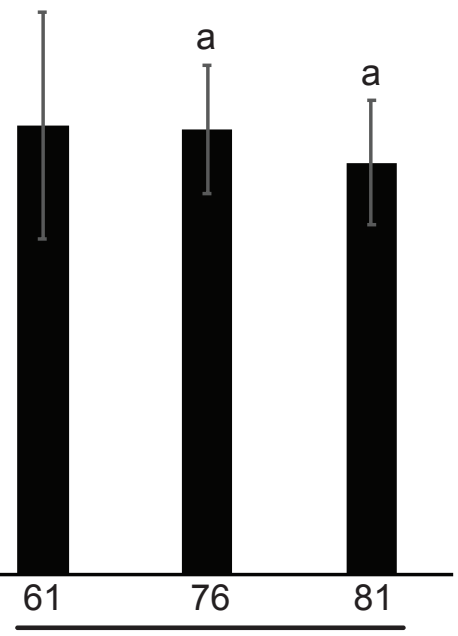

Sha BnGLO1+AIARC1 $+A / S R K+A / S C R$ 
4

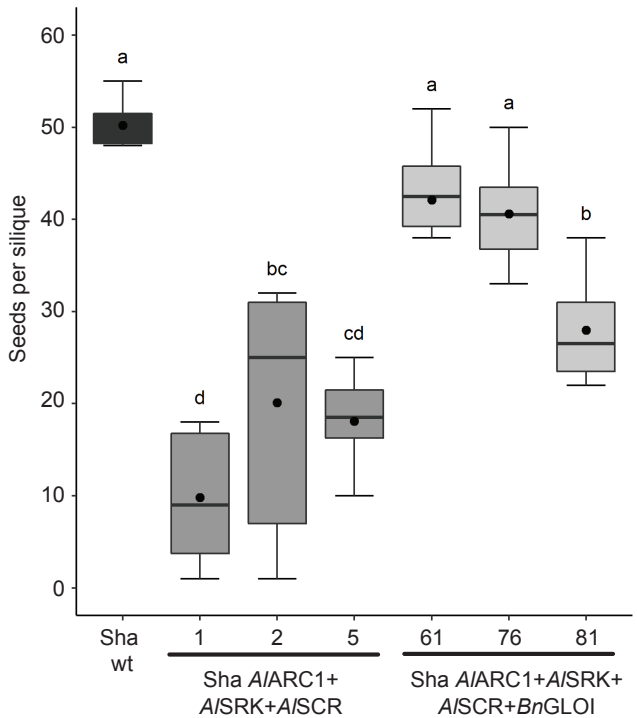


